\title{
EL CONSUMO DE INFORMACIÓN EN LA INDUSTRIA FARMACÉUTICA. II. RESULTADOS DE UNA ENCUESTA
}

\author{
María José Ordóñez Vergara*
}

Resumen: Continuando el estudio sobre el consumo de información de la industria farmacéutica, se exponen en este artículo los principales resultados del análisis estadístico de los datos obtenidos con un cuestionario dirigido a los laboratorios farmacéuticos ubicados en la Comunidad de Madrid, con la finalidad de recabar información sobre las necesidades y hábitos de información de este colectivo industrial. Para la realización del estudio estadístico se procedió al establecimiento de tipologías de encuestados, a la vez que de empresas atendiendo a diferentes parámetros como la actividad principal desarrollada dentro de la Comunidad de Madrid, el volumen de plantilla de las empresas, la nacionalidad de las mismas o la utilización de servicios de información.

Palabras clave: estudios de usuarios, necesidades y hábitos de información, consumo de información, bibliometria, industria farmacéutica, cuestionario.

\begin{abstract}
Continuing with the study of information consumption in the pharmaceutical industry, this article describes the main results of the statistical analysis of the data obtained with a questionnaire completed by the pharmaceutical laboratories in the Madrid Region, in order to gather data on the information needs and habits of this industrial sector. In order to prepare the statistical study, typologies were established for those who completed the questionnaire and for companies, applying different parameters, such as the main activity carried on in the Madrid Region, the companies' headcount, their nationalities or the use of information services.

Keywords: studies on users, information needs and habits, use of information, bibliometrics, pharmaceutical industry, questionnaire.
\end{abstract}

\section{Introducción}

Es un hecho conocido que la información se ha consolidado como un elemento indispensable de la gestión empresarial, fundamental para la adopción de nuevas estrategias como en cualquiera de las demás actividades de la empresa. Asimismo, se puede afirmar que la empresa mejor informada será la más competitiva, y por ende, aquélla que pueda acceder más rápidamente a la información que precisa, estará en mejores condiciones de reaccionar a los cambios de la demanda. Por ello los objetivos del estudio realizado se establecen en torno a una premisa fundamental: la utilización de información se configura como herramienta esencial para el desarrollo industrial. Una información veraz, completa y actual se constituye en el soporte de toda empresa competitiva y flexible.

* Universidad de La Coruña.

Recibido: 1." versión: 10-1-00; 2." versión: 24-4-(0). 
El proceso de concentración que experimenta desde principios de la década de los 90 el sector farmacéutico hace que esta situación se acentúe. En un entorno de «mercado global», el acceso y la utilización de información se convierte en elemento decisivo para la obtención y el mantenimiento de cuotas de mercado y, en definitiva, para la supervivencia de la propia empresa; máxime cuando se trata de sectores industriales que dependen fuertemente de la investigación para su desarrollo, como es el caso del sector farmacéutico. Avalando esta aseveración, los recientes resultados de la encuesta sobre «Innovación tecnológica en las empresas. 1998» realizada por el Instituto Nacional de Estadística (INE) y publicada en diciembre de 1999, según la cual el sector farmacéutico es el segundo en España en innovación (46\% de las empresas), después del sector petrolero (53\%). Los gastos en innovación corresponden a actividades de I+D y a otras actividades innovadoras entre las que destaca la adquisición de maquinaria y equipo relacionados con nuevos productos y procesos. Señala, además, que el sector farmacéutico dedica a innovación un $5 \%$ de su cifra de negocios.

Este segundo acercamiento a la realidad tocante a la información de las empresas farmacéuticas de la Comunidad de Madrid (en adelante CM) se obtuvo mediante un estudio de campo realizado en el año 1996 sobre una muestra elegida de la población total de empresas farmacéuticas de la Comunidad de Madrid. Basándonos en el censo de empresas anteriormente elaborado - previa revisión y actualización del mismo-, se agruparon los laboratorios atendiendo a diferentes criterios como: tamaño de las empresas, nacionalidad, actividades principales realizadas en la CM, etc., con la finalidad de escoger una muestra representativa. Permitiría, a la vez, estudiar por separado cada una de las tipologías establecidas. Asimismo, se determinó una serie de perfiles profesionales a los que dirigirnos dentro de dichas empresas.

Existe un trabajo pionero de finales de 1983 realizado conjuntamente por la Dirección General de Innovación Industrial y Tecnología del Ministerio de Industria y Energía y Fuinca (1) sobre necesidades de información de la industria española. Ya en ese estudio se procedió a una distinción horizontal y vertical dentro del análisis realizado. Es decir, se diferenciaba dentro de cada sector industrial según dimensión y actividad de la empresa, y en cada una de las empresas se realizaba un examen atendiendo a diferentes niveles de responsabilidad.

La recogida de datos en esta parte de la investigación se efectuó, básicamente, mediante los siguientes instrumentos: un formulario de entrevista y un cuestionario de preguntas cerradas.

El estudio estadístico realizado a partir de los datos obtenidos mediante las herramientas antes mencionadas, es representativo no sólo de la muestra de empresas elegida - según el muestreo por conveniencia adoptado - sino que los resultados son extrapolables, en líneas generales, al sector farmacéutico de ámbito nacional, ya que tal como se planteó el estudio, contemplando tanto los laboratorios farmacéuticos propiamente dichos como las delegaciones comerciales ubicadas en la CM y considerando tan sólo los dos grandes polos de concentración de las empresas de este sector en España (Madrid y Barcelona), el número de empresas farmacéuticas en el período estudiado era mayor dentro de la Comunidad de Madrid. 


\section{Metodología}

Mientras que para la recogida de datos en la primera fase del estudio realizado se recurrió a un método indirecto como es el análisis de peticiones de documentos (en concreto el examen de la demanda de información realizada por distintas empresas farmacéuticas ubicadas en la CM, y usuarias habituales del CINDOC), para esta parte de la investigación se decidió la utilización de métodos directos: la entrevista personal y el cuestionario por correo (para aquellos casos en los que no fue posible entrevistar personalmente), con el fin de completar la información sobre las necesidades y los hábitos de información que manifestaban las propias empresas.

Con la aplicación de métodos directos de recogida de datos, se pretendía depurar los resultados obtenidos mediante el empleo del método anterior, y obtener así una perspectiva más real sobre la problemática en cuanto a información presente en las empresas estudiadas. Por razones de operatividad, se estableció una limitación geográfica del estudio al ámbito de la Comunidad de Madrid ya que la viabilidad de las entrevistas personales en las empresas, previstas para esta segunda fase, hubiese sido muy costosa en el caso de abarcar otras áreas geográficas. En este sentido, tenemos que decir que se ha conseguido entrevistar a un número representativo de empresas (más del $50 \%$ de la población estudiada). Además, se buscó entrevistar al $100 \%$ de las empresas que desarrollaran actividades de $\mathrm{I}+\mathrm{D}$ dentro de la $\mathrm{CM}$, ya que en principio pensábamos que éstas podían ser las que más información necesitasen y utilizaran.

Aunque en principio, por razones económicas y de tiempo, podría haber parecido más efectivo enviar el cuestionario por correo al $100 \%$ de la población, y para la realización de las entrevistas personales determinar una muestra reducida de empresas, dada la índole de los usuarios estudiados, y con el fin de asegurar la obtención de un porcentaje de respuestas por parte de las empresas estadísticamente significativo de la población estudiada, se estimó oportuno solicitar dicha entrevista personal al $100 \%$ de las empresas farmacéuticas de la $\mathrm{CM}$, llevándola a cabo en aquellos casos en que accedieron a ello, y procediendo a enviar el cuestionario por correo a las restantes empresas.

La aplicación de esta metodología de investigación sobre un colectivo industrial concreto en nuestro país es probablemente la parte más novedosa de este trabajo, ya que no son numerosos los estudios de usuarios realizados en colectivos industriales determinados basados en métodos directos (2). Presenta ventajas tales como aumento de la tasa de respuesta, mayor fiabilidad de los datos y conocimiento directo (in situ) de las necesidades concretas de información de los usuarios; no estando exento de inconvenientes como la dificultad para conseguir las citas para llevar a cabo las entrevistas y un mayor coste presupuestario (gastos en desplazamientos, llamadas telefónicas, etc.).

La entrevista personal se realizó, siempre que fue posible, a los responsables de los servicios de información de los laboratorios o, en su defecto, a directivos o investigadores de dichas empresas, informándoles de los objetivos del trabajo y pidiéndoles información general sobre su empresa y sobre el modo cómo actualmente gestionan la información, valiéndonos para ello de un formulario semiestructurado de preguntas abiertas.

La ventaja fundamental de la estructuración de la entrevista ha sido la de garantizar que cuestiones importantes no quedasen sin tratar. Las preguntas planteadas en el formulario de entrevista hay que entenderlas como recurso para el entrevistador -que 
impida el olvido de temas relevantes-, más que como cuestiones a formular literalmente al entrevistado. De esta forma el entrevistador tiene la posibilidad de matizar, profundizar u omitir las diferentes cuestiones, según lo estime oportuno. Como es de suponer, no en todas las entrevistas personales realizadas fue posible proceder de igual forma, ya que las circunstancias para cada una de ellas variaban dependiendo del tiempo disponible por parte del entrevistado y de las facilidades dadas por éste para proseguir con la estructuración prevista.

En la entrevista personal, además de dar respuesta a una batería de preguntas abiertas (siguiendo el formulario de entrevista diseñado), se cumplimentó un cuestionario de preguntas cerradas (el mismo que fue enviado por correo a las empresas no entrevistadas personalmente). Por ello, hay que concebirla indisociablemente unida a la cumplimentación del cuestionario. Y aunque la entrevista personal estaba estructurada y articulada en su desarrollo mediante la utilización de un formulario diseñado a tal fin, en todos los casos para cerrar la misma se le pedía al entrevistado que rellenase el cuestionario de preguntas cerradas. Sólo si el tiempo concedido para la entrevista era escaso, se comenzaba por el cuestionario de preguntas cerradas. Luego, si era posible, se ampliaban temas mediante el formulario de entrevista, que servirían como preguntas de «control» para comprobación de la veracidad de las respuestas dadas en el cuestionario, o para su matización.

Una vez alcanzada la meta de entrevistar personalmente a más del $50 \%$ de la población estudiada, se completó la recogida de datos en esta segunda etapa de la investigación mediante el envío del cuestionario por correo a aquellas empresas de las que aún no se tenían datos, abarcando de ese modo la población total de empresas farmacéuticas de la CM. La incidencia de respuestas por correo ha sido escasa, aproximadamente el $12 \%$ del total de empresas censadas, confirmándose uno de los principales inconvenientes que supone su aplicación: el bajo porcentaje de respuesta. Así, se ha comprobado que en la utilización de este método es imprescindible, una vez enviado el cuestionario, hacer un seguimiento telefónico para comprobar su correcta recepción y averiguar qué empresas tienen intención de cumplimentarlo. En nuestro estudio, de aquellas empresas que accedieron a cumplimentarlo, se hizo un seguimiento telefónico hasta su devolución.

El esquema de trabajo diseñado para esta fase de recogida de datos mediante la técnica mixta de entrevista personal y encuesta por correo fue el siguiente:

- actualización del censo de empresas farmacéuticas de la CM;

- pequeño estudio de los tipos de usuarios de información a los que nos íbamos a dirigir y establecimiento del método y estrategia idóneos para recabar la información de interés para el estudio;

- depuración del censo de empresas mediante consulta telefónica (dirección actual de la empresa y persona de contacto);

- diseño preliminar del cuestionario y de la entrevista; discusión con expertos;

- envío de cartas de presentación a todas las empresas del censo, solicitando entrevistas personales;

- diseño del cuestionario, entrevistas piloto y prueba del cuestionario en varias empresas que atendieron nuestra petición;

- diseño final del cuestionario y preparación de todos los cuestionarios a utilizar;

- localización geográfica de las empresas; 
- concertar citas con empresas, realización de entrevistas personales (37 entrevistas efectuadas por un único entrevistador);

- envío de cuestionarios por correo a aquellas empresas no entrevistadas personalmente;

- unificación de preguntas comunes de aquellos cuestionarios correspondientes a empresas en las que se ha entrevistado a más de una persona;

- clasificación de los cuestionarios por categorías (dimensión, actividad, nacionalidad, etc.) y perfiles profesionales (documentalista, directivo, investigador);

- informatización de los cuestionarios atendiendo a los criterios anteriormente establecidos;

- tabulación de los datos;

- análisis estadístico en total, según las diferentes categorías establecidas y por perfiles profesionales; elaboración de gráficos;

- estructuración, tabulación y análisis de preguntas abiertas.

Se confeccionaron dos cuestionarios diferentes:

- uno para el caso de que el destinatario o entrevistado fuese un documentalista, encargado del suministro de información o persona con ese cometido dentro de la empresa;

- otro cuestionario para directivos e investigadores (usuarios-demandantes de información).

Sirva esta distinción aunque, por supuesto, el documentalista también es usuario-demandante de información, por ejemplo al consultar o utilizar fuentes o servicios de información externos.

Asimismo, se diseñaron tres tipos diferentes de formularios de entrevista para:

- documentalista;

- directivo, e

- investigador.

Clasificar a cada una de las personas entrevistadas y/o encuestadas -en correspondencia con los tres perfiles profesionales mencionados- no fue fácil en todos los casos, ya que a veces éstas no ejercían una función única dentro de la empresa. A efectos del estudio se optó por encuadrarlos en lo que parecía su «papel» fundamental o más determinante.

Al igual que los cuestionarios, los diferentes modelos de formularios de entrevista diseñados contenían unas preguntas comunes y otras específicas, atendiendo a los perfiles profesionales previstos, intentando recoger las características más significativas del comportamiento de cada uno de ellos con respecto a la información. A la vez permitieron un tratamiento de los datos más objetivo, al posibilitar controlar el sesgo que significaba cada uno de esos diferentes perfiles de personas entrevistadas $\mathrm{y} / \mathrm{o}$ encuestadas. 


\section{Resultados}

Como decíamos al principio, la finalidad de este estudio estadístico ha sido conocer las necesidades, tanto reales como potenciales y los hábitos de información de los laboratorios farmacéuticos de la CM, a partir del análisis estadístico de los datos obtenidos mediante un cuestionario de preguntas predefinidas a las que ha respondido una muestra representativa de las empresas.

El cuestionario fue cumplimentado por un total de treinta y nueve empresas, que suponen el $61 \%$ de los laboratorios farmacéuticos ubicados en la Comunidad de Madrid.

Se trata de laboratorios farmacéuticos en los que se dan actividades de producción, investigación, desarrollo, comercialización y distribución (en algunos todas, en otros sólo algunas de ellas) exclusivamente destinados al consumo humano; y con un volumen de empleados (plantilla de estas empresas en España, según los datos resultantes de la consulta de diversos directorios de empresas, y de las visitas a los laboratorios) muy dispar, de 4 (este número corresponde a los empleados de plantilla. La red de ventas, en este caso concreto, está formada por comerciales que no son empleados fijos de la empresa, luego no los incluimos), 10, 20, 20, 25, 65, 71, 90, 100, 120, 125, 150, $155,180,200,205,217,220,240,250,260,270,300,336,350,375,386,420,447$, $450,470,575,600,600,622,700,900$, y dos de 1500 empleados. Así, tenemos desde empresas familiares nacionales que cuentan con casi 100 años desde su creación y no han evolucionado mucho, a representaciones de multinacionales que tienen una supremacía a nivel mundial, siendo predominante la gran y mediana empresa multinacional extranjera.

De acuerdo con los fines de nuestro estudio, se procedió a un análisis en profundidad de las empresas atendiendo a los diferentes parámetros establecidos. Las características más relevantes, respecto al tamaño (según el número de empleados de plantilla) y la nacionalidad de los laboratorios sobre los que hemos realizado la investigación, se muestran en el siguiente cuadro (Tabla I).

Tabla I

Tamaño y nacionalidad de las empresas

\begin{tabular}{|lc|c|c|c|c|}
\cline { 2 - 6 } \multicolumn{1}{c|}{} & Grande & Mediana & Pequeña & Microempresa & TOTAL \\
\hline Nacionales & 2 & 4 & 3 & 1 & 10 \\
\hline Extranjeras & 16 & 9 & 1 & & 26 \\
\hline Mixtas & 2 & 1 & & & 3 \\
\hline Total $^{\prime}$ & 20 & 14 & 4 & 1 & 39 \\
\hline Porcentaje & $51,3 \%$ & $35,9 \%$ & $10,2 \%$ & $2,6 \%$ & $100 \%$ \\
\hline
\end{tabular}

Respecto al tamaño, la agrupación se realizó en base al texto de la «Recomendación de la Comisión Europea de 3 de abril de 1996 sobre la definición de pequeñas y medianas empresas» (Diario Oficial de las Comunidades Europeas. N. ${ }^{\circ}$ L 107/4-9 ES. 30-4-96), donde se establece: 
- grandes empresas, aquéllas que tienen más de 250 empleados en su plantilla;

- medianas, las de más de 50 empleados y menos de 250;

- pequeñas, aquéllas con más de 10 empleados y menos de 50;

- microempresas, las que emplean a menos de 10 personas.

En cuanto a la nacionalidad, consideramos tres categorías principales de laboratorios farmacéuticos:

- nacionales, empresas de capital español;

- extranjeros, aquellas empresas filiales de compañías no españolas;

- mixtos, las empresas resultantes de fusiones entre compañías de varios países, entre ellos España. A efectos de recuento de las empresas consideradas mixtas prevalecía la nacionalidad del país donde hubiese mayor número de accionistas.

Atendiendo a la actividad principal en la que los hemos encuadrado, se obtiene una nueva clasificación que vemos en la Tabla II.

Tabla II

Tamaño y actividad principal de las empresas

\begin{tabular}{|l|c|c|c|c|}
\cline { 2 - 5 } \multicolumn{1}{c|}{} & Producción & Investigación básica & Investigación clínica & TOTAL \\
\hline Grande & 2 & 10 & 8 & 20 \\
\hline Mediana & 6 & 3 & 5 & 14 \\
\hline Pequeña & 3 & & 1 & 4 \\
\hline Microempresa & 1 & & 14 & 1 \\
\hline Total & 12 & 13 & $35,9 \%$ & 39 \\
\hline Porcentaje & $30,8 \%$ & $33,3 \%$ & $100 \%$ \\
\hline
\end{tabular}

Para ello, establecimos las actividades más relevantes que podían desarrollar las empresas farmacéuticas de la CM: producción, investigación (básica y clínica), y comercialización-distribución. Atendiendo al censo de «empresas farmacéuticas de la Comunidad de Madrid», previamente elaborado, teníamos firmas con alternancia de todas estas actividades, de algunas o de sólo una de ellas. La única actividad que se da con exclusividad en determinadas empresas de la $\mathrm{CM}$ es la de comercialización-distribución de productos farmacéuticos; ésta determinará la radical diferencia entre delegaciones comerciales y laboratorios farmacéuticos.

Habría que hacer una matización más: en este trabajo utilizamos «investigación básica» no en el sentido estricto del término (aquella exclusivamente dirigida a ampliar el conocimiento científico, sin buscar ningún fin práctico determinado) sino en contraposición a «investigación clínica». Es decir, lo que aquí llamamos investigación básica es, prácticamente en todos los casos dentro de la industria farmacéutica, «investigación aplicada" ya que se persiguen fines concretos: desarrollo de nuevos productos (fármacos), nuevas aplicaciones, etc. Ello permitió realizar una distinción fundamental para nuestro estudio del tipo de investigación llevada a cabo por los diferentes laboratorios estudiados. No se excluye que en determinadas investigaciones realizadas por laboratorios farmacéuticos se den, además, componentes de investigación básica propiamente dicha. 
Con referencia a los perfiles profesionales en los que se ha clasificado a las personas entrevistadas y/o encuestadas - en relación al cometido principal desempeñado por cada una de ellas dentro de la empresa - corresponden en un $40,6 \%$ a encargados de búsquedas de información (fundamentalmente bibliográficas), responsables del servicio de información bibliográfica o servicio de documentación. Un $35,1 \%$ a investigadores y el $24,3 \%$ restante a personas que ejercen dentro de su departamento, principalmente funciones directivas, independientemente del departamento del que se trate. Por ello a efectos del estudio los hemos considerado como «documentalistas», «investigadores» y «directivos».

En general, se puede afirmar que la entrevista con directivos en todos los casos aportó una visión global de la organización, máxime en el caso de miembros de la Alta Dirección de la empresa. Además, reportó datos sobre otros tipos de informaciones, que ampliaban y complementaban las necesidades de información que puede tener este colectivo industrial. La óptica dada por los documentalistas estuvo, generalmente, más circunscrita a su ámbito de trabajo. Su visión de las necesidades de información dentro de la empresa suele estar bastante sesgada y deformada profesionalmente por su propio cometido, por lo que su aportación se centra en declaraciones sobre herramientas y soportes de información, y se restringe casi exclusivamente a las necesidades de información científica. En la mayoría de los entrevistados se comprobó que carecían de una perspectiva general de la organización, de lo que se hace en otros departamentos, etc. Algo similar ocurre con los investigadores.

De ahí que lo que en principio podría haber sido considerado como un error metodológico, a saber, no restringir el perfil profesional a entrevistar/encuestar, posibilitó en cambio la obtención de perspectivas diferentes sobre la problemática en relación a la información de estas empresas; lo que ha redundado en una mayor riqueza de matices y amplitud del análisis realizado.

Inicialmente, en el programa de trabajo de recogida de datos, se estimó una duración aproximada para las entrevistas de 25-30 minutos. El tiempo real concedido por cada una de las empresas entrevistadas ha ido de una a cuatro horas, con un promedio de dos horas por entrevista.

El promedio aproximado de edad de las personas entrevistadas/encuestadas incluidas en el perfil profesional de documentalista es de 38 años; en el de directivo de 39 años y en el de investigador de 44 años.

El nivel académico del perfil profesional directivo es el de licenciado - sólo un responsable de departamento comercial sin estudios universitarios--, donde se dan cabida diversas licenciaturas: Farmacia, Medicina, Económicas, etc. Tuvimos entrevista personal con dos altos directivos pertenecientes a la Dirección General de la empresa (un Director General y un Director Adjunto). Los restantes fueron Directores Técnicos, de Marketing, Comercial, Comunicación y relaciones externas, etc. Se han efectuado entrevistas personales con directivos, en empresas grandes, medianas y pequeñas, tanto nacionales como filiales de multinacionales extranjeras.

El perfil académico de los encargados de los servicios de información y documentación entrevistados (66,6\% de ellos, mujeres) corresponde mayoritariamente (a excepción de cuatro doctores en Medicina, Ciencias Químicas y Ciencias Biológicas) al de licenciado en Ciencias Químicas, Medicina, Farmacia y Ciencias Biológicas (nombrados según licenciaturas predominantes), tan sólo uno de ellos con formación humanística. El cargo que ocupan los entrevistados encuadrados profesionalmente como do- 
cumentalistas dentro de las grandes empresas es, -exceptuando un Director Médico-, el de Gerente (encargado, jefe) del servicio de documentación o servicio de información bibliográfica (3). Estos servicios son dependientes presupuestariamente -en la mayoría de los casos examinados- del Departamento de Investigación o del Departamento Médico de la empresa. En las medianas y pequeñas empresas, los responsables de búsquedas de información entrevistados ocupan el cargo de Director Médico o personal técnico, ejerciendo una doble labor dentro de sus respectivos departamentos. En las pequeñas empresas también encontramos técnicos (dentro de los Departamentos Técnicos) que ejercen de manera no formal las tareas de búsqueda de la información que necesitan. En la mayoría de los casos se trata de búsquedas de información científica, tanto bibliográfica como del propio documento.

Todos los doctores en Ciencias - a excepción de cuatro, responsables del Servicio de Documentación en grandes empresas - se encuentran dentro del perfil profesional de investigador. El cargo que ocupan en la empresa es el de Director de Investigación $(69,3 \%)$, Director Médico (23\%) y Director Técnico $(7,7 \%)$ y se sitúan en los respectivos Departamentos de I+D, Departamento Médico y Departamento Técnico de grandes y medianas empresas, principalmente filiales de multinacionales extranjeras.

A continuación, apuntamos los resultados más significativos una vez realizado el análisis estadístico - añadiendo gráficos ilustrativos - con los datos obtenidos de los laboratorios encuestados:

a) Con relación a las actividades principales (figura 1), vemos que son las de comercialización-distribución de productos farmacéuticos, así como la producción de los mismos, las actividades más desarrolladas en la Comunidad de Madrid. Le siguen en importancia las actividades de investigación clínica, realizadas en cooperación con los principales hospitales de la CM. Las actividades de investigación básica se realizan en menor porcentaje. Es de suponer que bastantes laboratorios filiales de multinacionales extranjeras desarrollen esta actividad en sus países de origen.

En este gráfico y en los siguientes hay que aclarar que las diferentes columnas no han de sumar $100 \%$, ya que los resultados de cada una de ellas no son excluyentes.

De los 39 laboratorios que componen la muestra estudiada, un $77 \%$ pertenece a "grupos» empresariales. El cuestionario nos ha permitido recoger datos sobre las actividades que desarrolla un $73 \%$ de éstos fuera de la Comunidad de Madrid. Según los resultados obtenidos se incrementan las actividades de investigación - fundamentalmente la investigación básica- y el desarrollo de nuevos productos, así como las de marketing y los estudios de mercado.

b) En cuanto a las necesidades de información, según declaran los propios laboratorios, tienen grandes necesidades de información de carácter científico y técnico, como vemos en el gráfico (figura 2).

Según manifiestan los documentalistas entrevistados, dentro de la organización los departamentos que más información solicitan son (en orden decreciente) los de: «Investigación», "Comercial/Ventas», "Dirección General/Finanzas", y «Producción/Control/Técnico». Ha sido necesario el agrupamiento de algunos departamentos ya que en muchos laboratorios no están claramente diferencia- 
Figura 1

\section{Actividades principales}

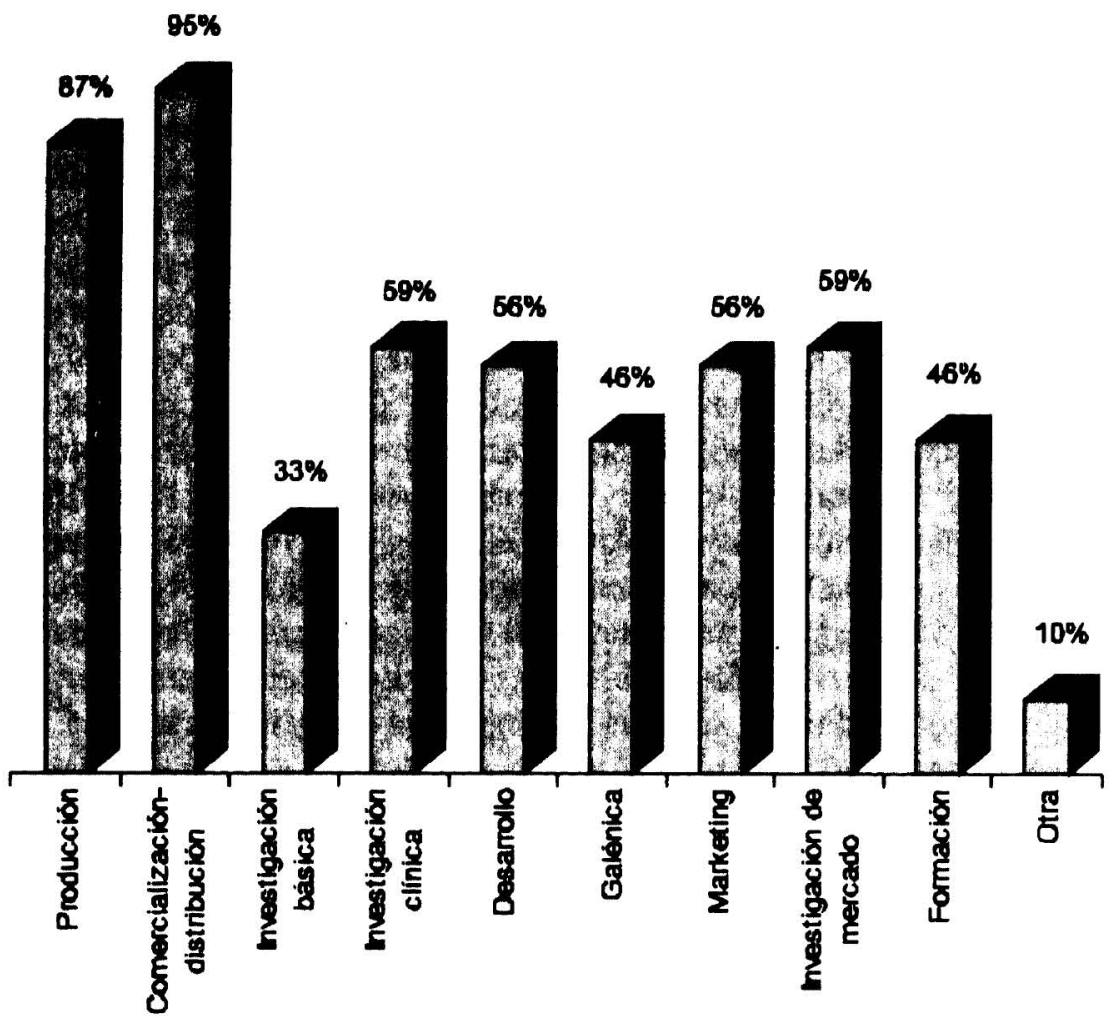

Figura 2

Necesidades de información

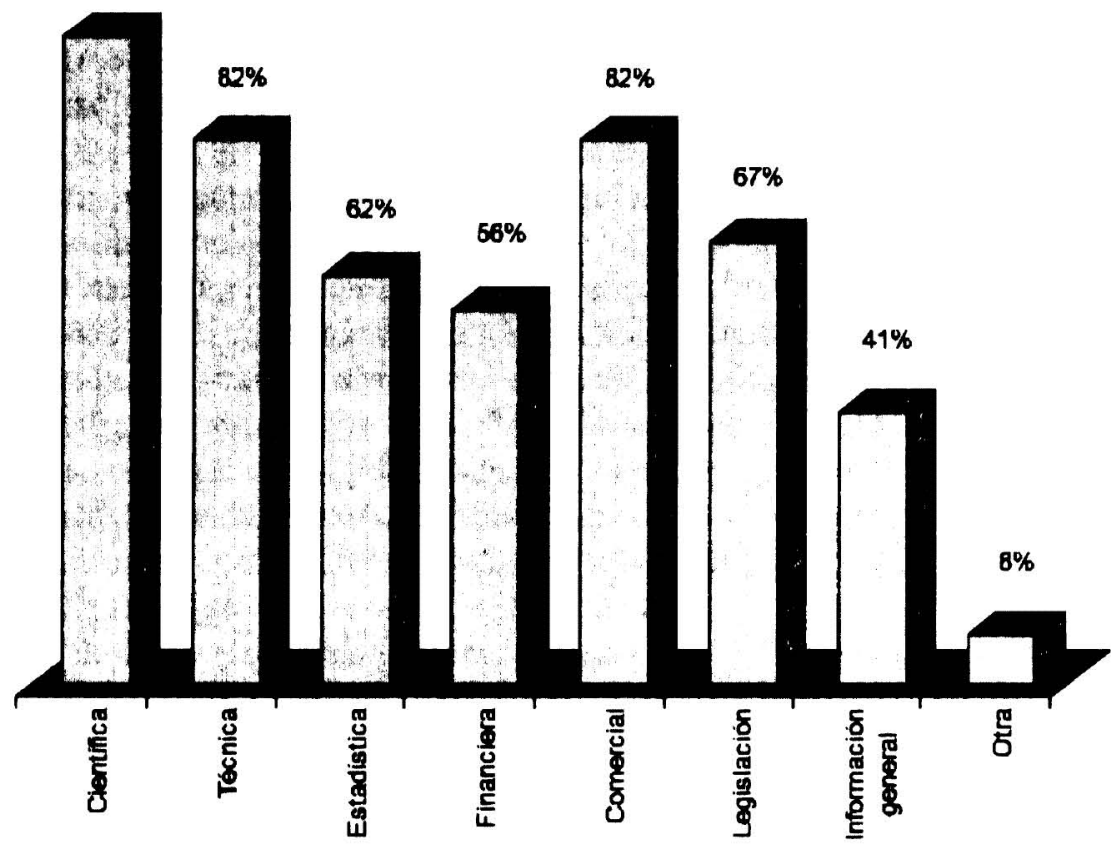


dos, produciéndose el solapamiento de algunos, sobre todo en la pequeña y mediana empresa. En todos ellos la información más solicitada - tal como apuntan los documentalistas- es la científica, seguida de la información comercial y técnica.

c) Con referencia al tipo de documento más utilizado, al igual que en la primera fase del estudio, se revela una mayor utilización de los artículos, obtenidos en revistas científicas y en bases de datos de biomedicina.

Es de destacar la poca utilización de tesis doctorales y patentes (ver figura 3). Las primeras por su difícil acceso, según palabras de los propios encuestados. Las segundas, estimamos, debido al reducido número de laboratorios dedicados a actividades de investigación dentro de la CM. Pese a ello, no deja de llamar la atención esta baja demanda ya que, en principio, este tipo de documento parece que debería ser prioritario para las empresas de este sector. Tal como señalan los investigadores entrevistados, para satisfacer estas necesidades de información recurren a empresas y organismos externos especializados en patentes. Principalmente a la Oficina Española de Patentes y Marcas (OEPM) dependiente del Ministerio de Industria y Energía.

Figura 3

Tipología documental

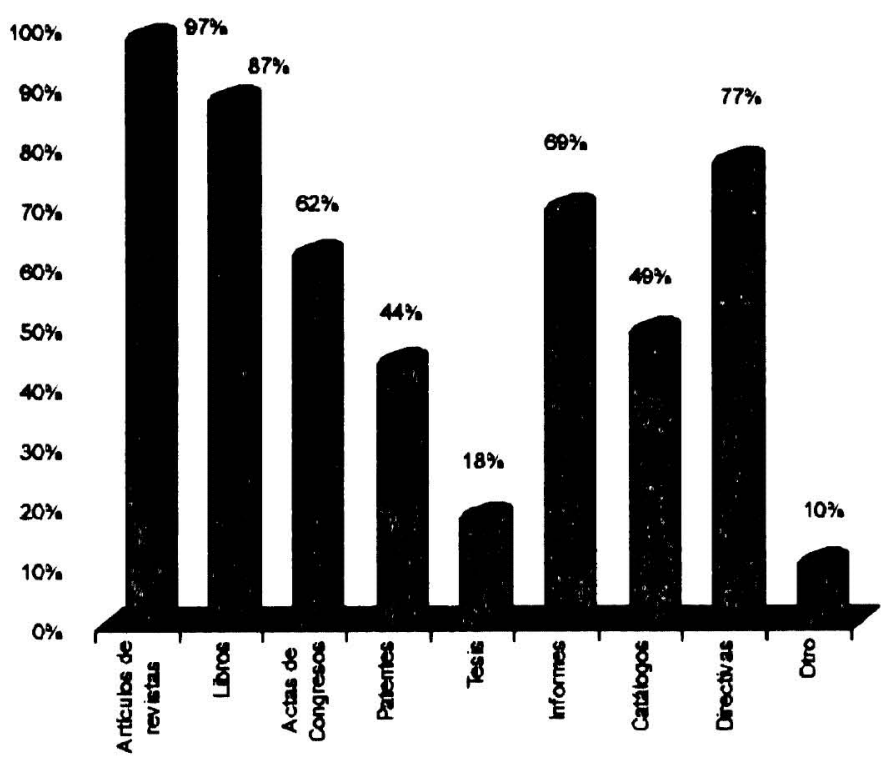

d) En relación a la procedencia de la información (figura 4), los laboratorios analizados dicen satisfacer sus necesidades de información, principalmente, a través de la información suministrada desde la casa matriz, de información y documentación interna, y por medio de centros de documentación: externos, e internos.

También es destacable la información obtenida a través de redes informáticas de comunicación, en muchas de las empresas encuestadas a partir de su red 
corporativa o intranet (en este porcentaje incluimos aquéllas que se estaban instalando en los laboratorios visitados en el momento de realizar el estudio).

\section{Figura 4}

\section{Procedencia de la información}

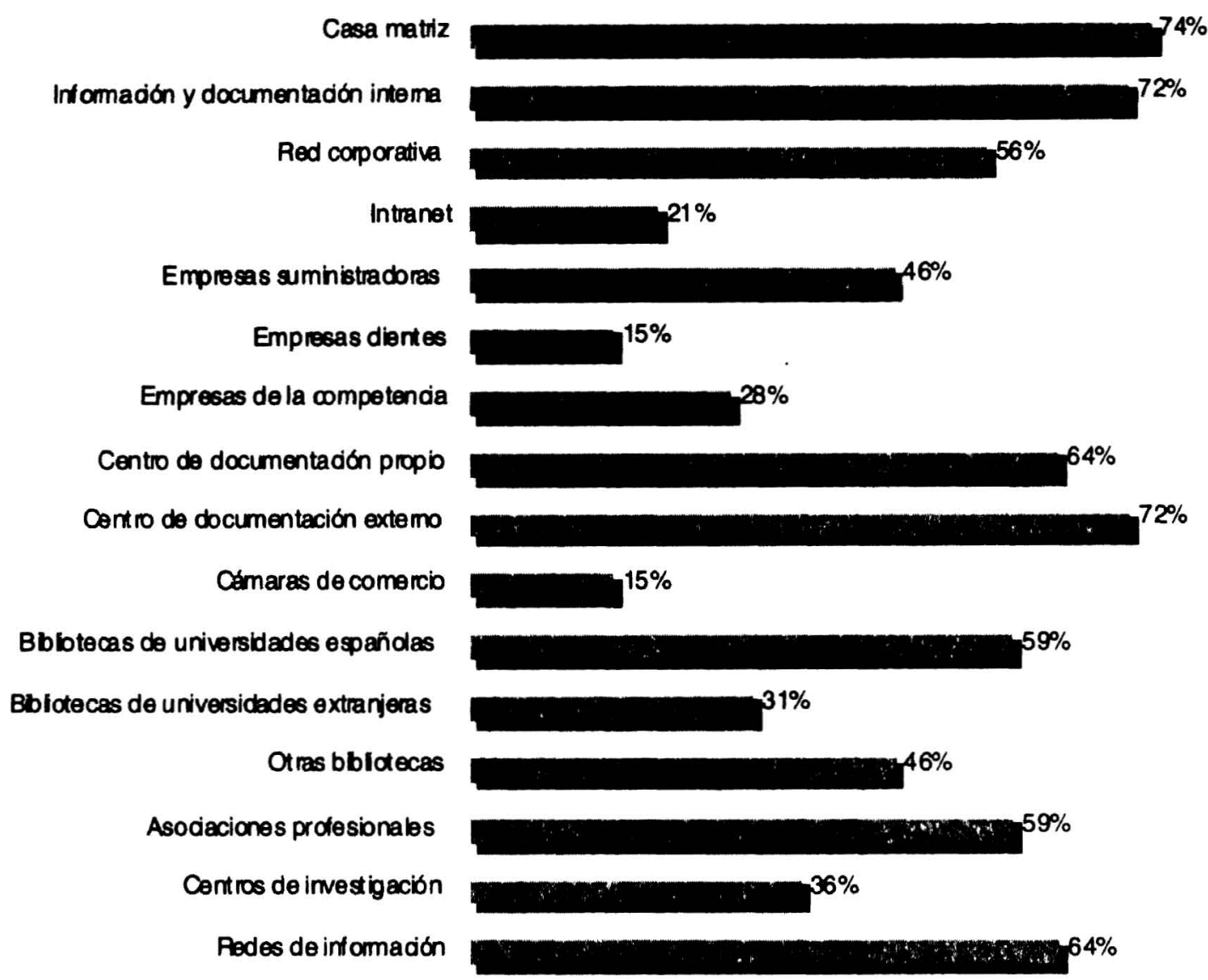

Hay que introducir matizaciones en este $64 \%$ obtenido de empresas que consiguen información a través de un centro de documentación propio, ya que de éstas tan sólo un $41 \%$ - lo que corresponde a 16 laboratorios- dispone de auténticos centros de información y documentación, con instalaciones propias y personal especializado; por personal especializado entendemos: conocedores de fuentes y herramientas informativas, e incluso aquéllos que han realizado algún cursillo en este campo, pero no encontramos en las empresas visitadas a ninguna persona con estudios universitarios específicos en Documentación, a excepción de dos jóvenes en prácticas, alumnos de Biblioteconomía y Documentación. El $23 \%$ restante cuenta con una pequeña biblioteca y alguna persona encargada de las búsquedas en el exterior (no tratándose en ninguna ocasión de especialistas en Información y Documentación); o, en el caso de algunas fíliales de multinacionales extranjeras, de centros de documentación situados en sus casas 
matrices. De cualquier manera, se trata de un porcentaje muy elevado, demostrando de esta forma la importancia que dichos laboratorios conceden a la obtención y utilización de información y confirmando la ya notoria tradición de este sector en el uso de la información dentro del ámbito industrial español (5).

El 72\% de los laboratorios, para satisfacer sus necesidades de información recurre a centros de documentación o servicios de información externos, entre ellos - según especificaron en los respectivos cuestionarios- tenemos: el CINDOC y la British Library (BL).

Los servicios de información externos más utilizados por documentalistas, atendiendo al número de veces que han sido mencionados, son: el CINDOC, la BL y las bibliotecas de hospitales (principalmente de la CM). Además, señalan otros servicios de información utilizados por ellos, como: las bibliotecas de universidades españolas, las asociaciones profesionales, las agencias especializadas en información, los centros de investigación nacionales y las empresas suministradoras de bases de datos.

Puede deducirse, a tenor de las respuestas registradas, que el grado de satisfacción de los usuarios de los servicios de información externos es alto; si bien la velocidad de respuesta sería uno de los aspectos a mejorar, ya que en ocasiones la falta de cierta información supone la paralización o ralentización del trabajo, según manifiestan los propios encuestados.

e) Con referencia a los soportes de información más utilizados, actualmente, por las empresas estudiadas, tenemos: el soporte papel seguido de los soportes magnéticos y ópticos (figura 5). En el futuro, según estimación de los propios laboratorios, la tendencia será hacia el incremento en el empleo de los soportes óptico y on-line, con un descenso del soporte papel.

Como vemos se tiende a una utilización cada vez mayor de soportes electrónicos (informáticos) con gran capacidad de almacenamiento, como es el caso de los soportes ópticos. Y al empleo generalizado de redes informáticas para la obtención y transmisión de datos e informaciones de forma inmediata, tanto internas como externas a la organización.

Tal como comentan los propios encuestados, les gustaría poder prescindir, cada vez más, del soporte papel y estar más informatizados. Pero añaden que, debido a la propia naturaleza de su sector, deben obligatoriamente tener gran parte de la documentación en soporte papel, fundamentalmente aquellos laboratorios que desarrollan actividades de investigación.

f) En cuanto a los deseos expresados con relación a la satisfacción de necesidades de información, aunque de manera global dicen estar satisfechos con la información que consiguen, parecen dispuestos — primordialmente los pequeños y los medianos laboratorios - a recibir propuestas sobre nuevos productos y servicios de información adecuados a sus propias necesidades.

- La primera necesidad manifestada son los «productos de información a medida» (55\% del total de laboratorios estudiados), sobre todo para la mediana y pequeña empresa nacional con producción y, especialmente, para directivos. En las entrevistas a investigadores se apunta la necesidad de disponer de productos de información más específicos a lo que cada uno necesita, como bases de datos adaptadas y orientadas a cubrir sus necesidades puntuales de in- 
Figura 5

Soportes de información

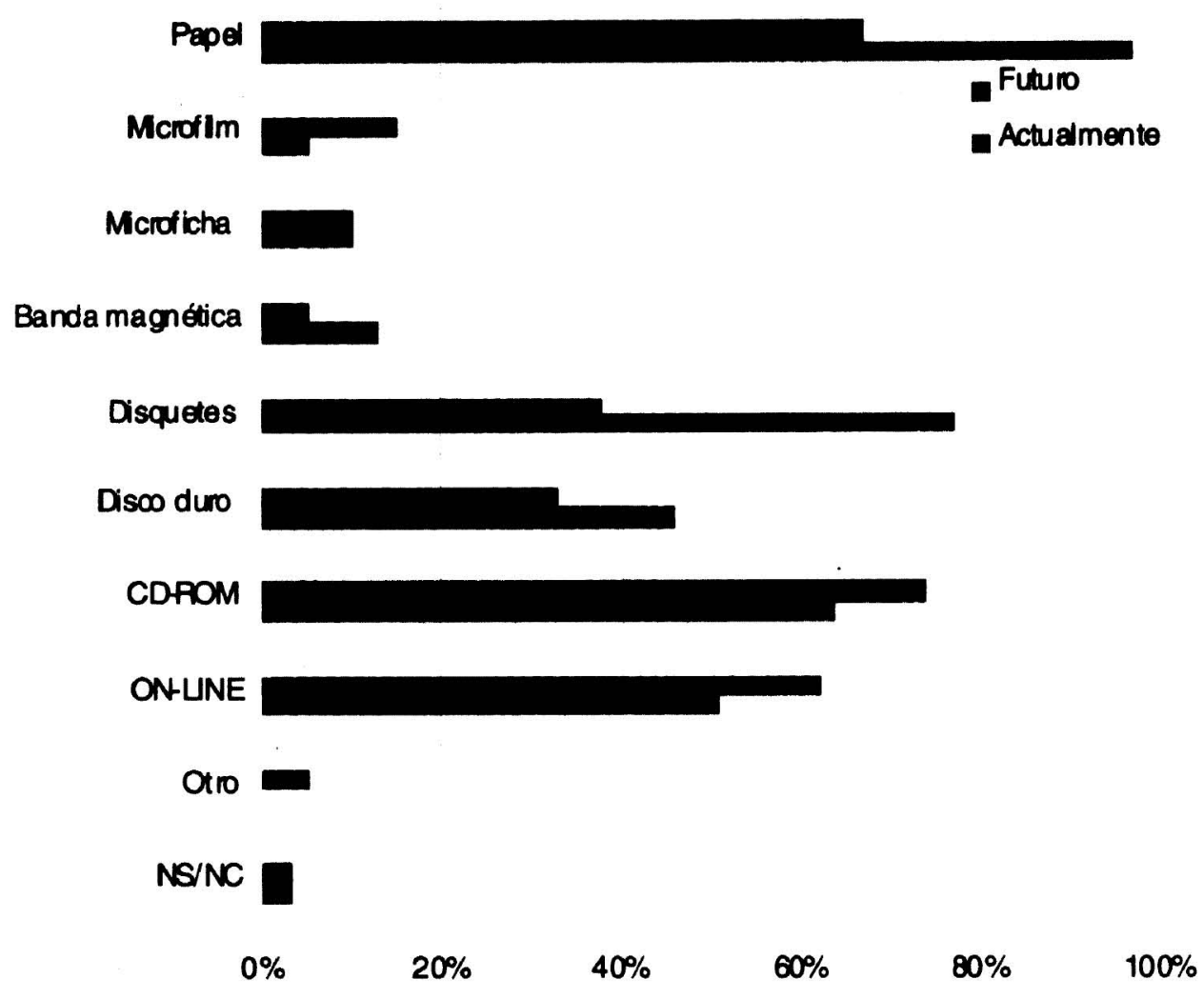

formación. Hemos constatado que algunos grandes laboratorios ya cuentan con bases de datos comerciales «personalizadas» (custom-made), conforme a necesidades concretas.

- Los «nuevos servicios de información» (50\%) son el segundo deseo manifestado por estos laboratorios, muy patente en aquéllos que tienen investigación y principalmente por directivos y documentalistas. Entre los nuevos servicios de información que señalan cabe destacar aquellos accesibles on-line, como Internet y correo electrónico. Los investigadores apuntan la rapidez de actualización como una de las grandes ventajas de la consulta de bases de datos y revistas electrónicas a través de Internet.

g) A la pregunta de si tenían un servicio de información interno (o, en su defecto, una persona encargada del suministro de información), el $82 \%$ de los laboratorios respondió afirmativamente, y estos son los resultados (hay que volver a insistir sobre el hecho de que los resultados no son excluyentes, ya que en algunos laboratorios se dan todas estas posibilidades e incluso el que cada cual realice sus propias búsquedas de información).

En el siguiente gráfico (figura 6) vemos lo expresado anteriormente: el $23 \%$ tiene un encargado de búsquedas, el $51 \%$ una biblioteca dentro de la empresa, el $41 \%$ un centro de documentación en la CM, y el $46 \%$ lo tiene fuera de la CM. 
Aunque sabemos que se dan algunos casos de colaboración entre algunos de los laboratorios entrevistados, la tónica general entre las empresas del sector farmacéutico es de «secretismo». Pero en la actualidad, por cuestiones de reducción de costes, algunas de ellas comparten centros de investigación y documentación científica, como pudimos constatar.

\section{Figura 6}

Procedencia de la información

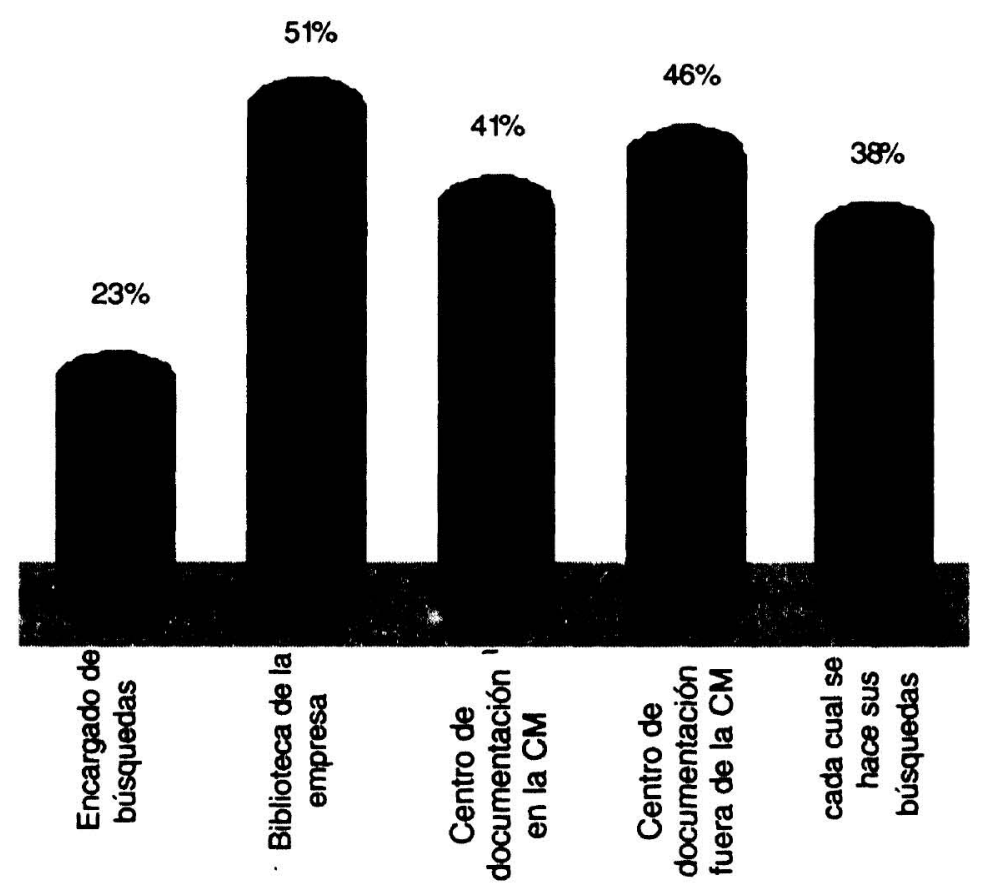

La creación de un centro de documentación que suministre información a toda la empresa le parece interesante (aquí sólo responden empresas que no tienen centro de documentación propiamente dicho) a un $36 \%$ de los laboratorios encuestados; mientras que un $23 \%$ no lo encuentra oportuno para su empresa. El $41 \%$ restante tiene centro de documentación en la CM.

Entre las opiniones a favor, destacaríamos:

- ahorro de tiempo que permitiría a los usuarios del servicio dedicarse exclusivamente a su propio trabajo, dejando que el centro de documentación resolviese sus problemas de información. A la vez contribuiría a la no duplicidad de esfuerzo en las búsquedas de información para diferentes sujetos;

- asesoramiento en las labores de búsqueda de información, así como en el uso y manejo de fuentes y herramientas informativas,

- obtención de información más completa y exhaustiva;

- actuaría de filtro para la selección y reparto de la información a cada departamento de la empresa. 
En general declaran que el exceso de información con el que cuentan actualmente se convierte en una de las principales barreras de acceso a la misma. Algunos apuntan que sólo el cribar la información que puede resultar de su interés les lleva gran parte de su tiempo de trabajo. Necesitan - según manifiestan- información más estructurada y filtrada.

\section{Conclusiones}

Entre las principales conclusiones a las que se ha llegado tras la realización del estudio cabe destacar que, en general, la investigación revela grandes diferencias en cuanto al nivel de utilización de la información como herramienta estratégica por parte de las empresas del sector farmacéutico.

A pesar de ello, se puede hablar (en general) de un nivel de utilización de información científica muy alto. El estudio confirma una relación directa entre la actividad desarrollada y las necesidades de información generadas. Es decir, las grandes necesidades de información científica en este sector se tienen cuando se llevan a cabo actividades de Investigación y Desarrollo.

Las revistas científicas y técnicas y la consulta de bases de datos biomédicas (principalmente en soporte CD-ROM) son las principales fuentes de información dentro del sector farmacéutico. El estudio demuestra una buena cobertura de las bibliotecas españolas cuando se trata de satisfacer la demanda de este sector.

Asimismo, se da una creciente utilización de medios electrónicos para consulta, almacenamiento, soporte y transmisión de información.

Con referencia a los hábitos de información, cabe destacar un predominio en el uso de canales informales por parte de los directivos; los cuales, declaran como una de sus principales fuentes de información los contactos telefónicos. Asimismo, los investigadores manifiestan que los contactos personales con colegas dentro de su campo de investigación son una importante fuente de información para ellos, aunque revelan un mayor conocimiento y utilización de canales formales. Aquéllos encuadrados bajo el perfil profesional de documentalistas consiguen información, principalmente, a través de canales formales, como las bases de datos y las revistas científicas.

Los resultados del estudio ponen de manifiesto un problema generalizado en la mayor parte de las empresas estudiadas: el exceso de información, y la pérdida de tiempo que conlleva seleccionarla, lo que es indicio del gran cometido a desempeñar en el ámbito industrial por los profesionales de la documentación para la búsqueda, selección, tratamiento y archivo de información.

Por el momento, hay una escasa utilización de la red Internet; aunque la mayoría declara considerarla una de las principales fuentes de información en el futuro. El correo electrónico y los foros de discusión son frecuentemente utilizados por los investigadores de laboratorios con actividades de Investigación y Desarrollo.

Tal como se desprende del estudio, del total de empresas que componen la población «sector industrial farmacéutico de la $\mathrm{CM}$ » (en el período estudiado) aproximadaménte el $19 \%$ cuenta con centros de documentación propiamente dichos. Estos se encuentran ubicados, mayoritariamente, en laboratorios filiales de multinacionales extranjeras. Estos servicios de información se dedican, casi exclusivamente, al suministro de información científica, dando fundamentalmente cobertura informativa a los 
Departamentos de Investigación, Departamento Médico y como apoyo documental para los delegados comerciales. Primordialmente, surten de información bibliográfica conseguida, sobre todo, a través de bases de datos biomédicas. Los documentos primarios los obtienen de servicios de información externos (CINDOC y BL, principalmente).

Según hemos constatado, el perfil académico del documentalista de laboratorio farmacéutico, por el momento, es de formación en áreas afines al sector en el que trabajan - como Medicina, Farmacia, Biología o Química- y cuentan con conocimientos sobre herramientas informativas y técnicas de documentación.

Parece que, debido fundamentalmente a la actual coyuntura económica, los departamentos de documentación de estas empresas son los primeros en sufrir recortes de presupuesto, cuando se trata de reducir costes. La tendencia actual es la de contratar servicios externos de información en el momento en que se necesitan. Con ello, se ahorra en instalaciones y personal. La velocidad de respuesta es uno de los parámetros más valorados a la hora de elegir centro de documentación externo.

Los medios más utilizados para las demandas de información continúan siendo los «clásicos»: fax, teléfono y peticiones realizadas personalmente. Excepto en el caso de las grandes empresas donde parece haber una mejor dotación tecnológica (sobre todo en aquéllas que desarrollan actividades de investigación), en las que el correo electrónico comienza a ser un medio «habitual» para la petición y transmisión de información.

En general, se puede hablar de una creciente cultura informativa en ámbitos industriales como el estudiado, y de ello es muestra el interés con el que las empresas colaboradoras han recibido el tema que les presentábamos. Lo que no hace más que confirmar la notoria tradición que el sector industrial farmacéutico tiene en el uso de información.

Por último, hay que señalar que, según se desprende de la investigación, es de vital importancia para el futuro de la profesión una adecuación entre las expectativas de los usuarios de información y lo que los especialistas de la información estamos suministrando.

\section{Referencias bibliográficas}

1. BERENGUER PEÑA, J. M. Hábitos y necesidades de información en las empresas industriales españolas. La información al servicio del empresario. Madrid: Cámara de Comercio e Industria de Madrid, 1986, pp. 95-110.

2. SANZ, E. y RUBIO, L. Necesidades de información en las empresas: estudio de un caso. Revista Española de Documentación Científica, 1993, vol. 16, n. ${ }^{\circ} 3$, pp. 229-236. -SANZ, E. «La realización de estudios de usuarios: una necesidad urgente». Revista General de Información y Documentación, 1993, vol. 3, n. ${ }^{\circ}$ 1, pp. 155-166.

3. RODRIGUEZ GAIRIN, J. M. Los servicios de información bibliográfica en Ciencias de la Salud. Estructura y organización. Actas 3as. Jornadas de Documentación e Información en Ciencias de la Salud. Sevilla, 1990, pp. 25-33.

4. JIMÉNEZ LÓPEZ, M. A. y GONZÁLEZ QUESADA, A. Dificultades de inserción laboral del bibliotecario-documentalista en el sector de la empresa privada: el caso de las industrias química y farmacéutica. $V$ Jornadas Españolas de Documentación Automatizada. Documat 96. Cáceres: FESABID, 1996, pág. 105.

5. SANZ, E.; HERNÁNDEZ, A.; ARAGÓN, I. y ÁlVAREZ, C. Study of scientific informa- 
tion needs of Spanish industry. IDT-93. Marchés et industries de l'information, l0ème Congrès. Paris, 1993.

6. BAIGET, T. Uso de información en laboratorios farmacéuticos. El Profesional de la información, 1999, vol. 8, n. ${ }^{\circ} 12$, pp. 15-21. 